\title{
7-IncRNA Assessment Model for Monitoring and Prognosis of Breast Cancer Patients: Based on Cox Regression and Co-expression Analysis
}

\author{
Huayao $\mathrm{Li}^{1 \dagger}$, Chundi Gao ${ }^{2 \dagger}$, Lijuan Liu ${ }^{3,4}$, Jing Zhuang ${ }^{3,4}$, Jing Yang ${ }^{3}$, Cun Liu' ${ }^{2}$, \\ Chao Zhou ${ }^{3,4}$, Fubin Feng ${ }^{3,4}$ and Changgang Sun ${ }^{5 *}$ \\ ${ }^{1}$ College of Chinese Medicine, Shandong University of Traditional Chinese Medicine, Jinan, China, ${ }^{2}$ College of First Clinical \\ Medicine, Shandong University of Traditional Chinese Medicine, Jinan, China, ${ }^{3}$ Department of Oncology, Weifang Traditional \\ Chinese Hospital, Weifang, China, ${ }^{4}$ Department of Oncology, Affiliated Hospital of Weifang Medical University, Weifang, \\ China, ${ }^{5}$ Chinese Medicine Innovation Institute, Shandong University of Traditional Chinese Medicine, Jinan, China
}

\section{OPEN ACCESS}

Edited by:

Aleix Prat,

Hospital Clínic de Barcelona, Spain

Reviewed by:

Luigi Formisano,

University of Naples Federico II, Italy

Chuanxin Wang

Second Hospital, Shandong

University, China

*Correspondence:

Changgang Sun

scgdoctor@126.com

${ }^{\dagger}$ These authors have contributed equally to this work and share first authorship

Specialty section: This article was submitted to

Women's Cancer, a section of the journal

Frontiers in Oncology

Received: 25 January 2019 Accepted: 15 November 2019 Published: 03 December 2019

Citation:

Li H, Gao C, Liu L, Zhuang J, Yang J,

Liu C, Zhou C, Feng F and Sun C

(2019) 7-IncRNA Assessment Model for Monitoring and Prognosis of

Breast Cancer Patients: Based on

Cox Regression and Co-expression Analysis. Front. Oncol. 9:1348. doi: 10.3389/fonc.2019.01348
Background: Breast cancer is one of the deadliest malignant tumors worldwide. Due to its complex molecular and cellular heterogeneity, the efficacy of existing breast cancer risk prediction models is unsatisfactory. In this study, we developed a new IncRNA model to predict the prognosis of patients with BRCA.

Methods: BRCA-related differentially-expressed long non-coding RNA were screened from the Cancer Genome Atlas database. A novel IncRNA model was developed by univariate and multivariate analyses to predict the prognosis of patients with BRCA. The efficacy of the model was verified by TCGA-based breast cancer samples. Identified IncRNA-related mRNA based on the co-expression method.

Results: We constructed a 7-IncRNA breast cancer prediction model including LINC00377, LINC00536, LINC01224, LINC00668, LINC01234, LINC02037, and LINC01456. The breast cancer samples were divided into high-risk and low-risk groups based on the model, which verified the specificity and sensitivity of the model. The Area Under Curve (AUC) of the 3- and 5-year Receiver Operating Characteristic curve were 0.711 and 0.734 , respectively, indicating that the model has good performance.

Conclusion: We constructed a 7-IncRNA model to predict the prognosis of patients with BRCA, and suggest that these IncRNAs may play a specific role in the carcinogenesis of BRCA.

Keywords: breast cancer, univariate and multivariate Cox analyses, bioinformatic analysis, 7-IncRNA model, co-expression analysis

\section{INTRODUCTION}

Breast cancer (BRCA) is considered as the leading cause of death among gynecologic neoplasias. The treatment of BRCA has markedly improved due to advances in early screening and the development of anticancer strategies (1). However, breast cancer still exhibits a high recurrence rate (2). Studies have shown that the prognosis of breast cancer is affected by many factors like 
age, tumor size, grade, lymph node involvement, lymphovascular invasion, histology, hormone-receptor status, c-erbB2 status, and positive margins (3). Due to the pathogenic complexity of breast cancer, although many breast cancer prognostic biomarkers have been discovered, prognosis remains a difficult problem $(4,5)$. There is a need to construct a new breast cancer risk prediction model to improve the treatment of breast cancer patients. Due to the gene signature is yet limited in coding genes and microRNAs, to prove the necessity to develop the lncRNA model for predicting BRCA survival.

In the post-genomic era, many genome sequencing techniques have emerged (6). These tools provide new ideas and insights for tumor diagnosis and prognosis prediction. These nextgeneration sequencing methods and the data can thereby help better identify clinical biomarkers of cancer. The discovery of long non-coding RNA (lncRNA) has dramatically altered our understanding of cancer. The expression and dysregulation of lncRNAs is more cancer-type specific than the protein-coding genes (7). The latest research shows that lncRNAs play key roles in gene regulation and carcinogenesis, including proliferation, adhesion, migration, and apoptosis (8). Given the heterogeneity of BRCA and the complexity of non-coding RNAs, a panel of lncRNA biomarkers may be more precise and stable for BRCA prognosis (9). Shi et al. (10), based on The Cancer Genome Atlas (TCGA) database, constructed a 31-lncRNA model, which might be able to predict Overall Survival (OS) in patients with lung adenocarcinoma with high accuracy. Long et al. (11), by integrating the high-throughput data from the TCGA database, screened four genes (CENPA, SPP1, MAGEB6, and HOXD9) using univariate, Lasso, and multivariate Cox-regression analyses to develop the hepatocellular carcinoma prognostic model.

In this study, we screened breast cancer-associated differentially-expressed IncRNAs from the TCGA database and developed a new lncRNA model to predict the prognosis of patients with BRCA. It is well-known that lncRNAs could affect the function of proteins and cells directly or indirectly due to their involvement in the regulation of mRNA (12). Therefore, we have further explored the function of lncRNA in the model by studying the function of lncRNA-related mRNA. In summary, the use of IncRNA features provides a deeper insight into the prognosis of BRCA, which may be helpful in guiding the treatment.

\section{MATERIALS AND METHODS}

\section{Data Source}

The lncRNA expression profiles and the corresponding clinical information from the patients with BRCA were obtained from The Cancer Genome Atlas (TCGA: https://cancergenome.nih. gov/) (13); a total of 1,208 samples, including 112 healthy and 1,096 BRCA samples. BRCA samples with incomplete prognostic

Abbreviations: LncRNAs, long non-coding RNAs; BRCA, breast cancer; OS, overall survival; TCGA, the Cancer Genome Atlas; GO, gene oncology; KEGG, Kyoto Encyclopedia of Genes and Genomes; ROC, receiver operating characteristic; AUC, Area Under Curve; BP, biological process; CC, cellular component; MF, molecular function. information were excluded, and the average expression level was used as the final expression data of the same patient mRNA and lncRNA. A total of 1,076 BRCA samples were selected for further construction of the prognostic risk model and co-expression analysis. As the information was retrieved from the TCGA database, a public database, further ethical approvals do not apply to our research. Data collection and processing are in line with TCGA data policies for protecting human subjects (http://cancergenome.nih.gov/publications/ publicationsguidelines).

TABLE 1 | Specific baseline clinical characteristic of 1,076 breast cancer patients.

1,076 breast cancer patients

Age

$<60$ years $\quad 572$

$\geq 60$ years $\quad 504$

Stage

॥ 610

III $\quad 244$

IV 19

Unknown 23

Pathologic T stage

T1-2

897

T3-4

176

Unknown

Pathologic N stage

NO-1

N2-3

862

Unknown

194

20

Pathologic M stage

MO

896

M1

Unknown

Estrogen receptor

Positive

790

Negative

237

Unknown

49

Progesterone receptor

Positive

683

Negative

341

Unknown

52

HER2

Positive

161

Negative

554

Unknown

361

Survival time

$\leq 1$ years

185

1 years <

482

$\leq 3$ years

3 years $<$

167

$\leq 5$ years

$>5$ years 


\section{Identification of Differentially-Expressed IncRNAs and mRNAs}

To identify the lncRNAs and mRNAs differentially expressed between the BRCA and the healthy samples, the downloaded lncRNA and mRNA data were standardized and differentialexpression analysis was performed using the edgeR software package in the $\mathrm{R}$ software. The lncRNAs and mRNAs were differentially expressed with an absolute $|\operatorname{logFC}|>$ 2 and $p<0.01$ were considered for subsequent analysis. The $\log \mathrm{FC}$ indicates the fold change in the expression of each IncRNA and mRNA between BRCA and healthy breast tissue samples. Volcano plot of the differentiallyexpressed lncRNAs and mRNAs was obtained using the R software.

\section{Definition of the IncRNA-Related Prognostic Model}

The IncRNA-related prognostic model was constructed based on the prognostic characteristics of lncRNA, and the correlation between overall survival (OS) and lncRNA expression levels was studied using univariate and multivariate Cox-regression analysis. Differences were assessed by univariate Cox proportional hazards regression analysis using $\mathrm{R}$ survival kits. For the association between expressed lncRNA and the overall survival, the lncRNA was considered significant when the $p$-value was $<0.01$ in the univariate Cox-regression analysis and was selected for multivariate Cox-regression analysis. Subsequently, multivariate Cox-regression analysis was performed to evaluate the contribution of genes as independent prognostic factors inpatient survival. A stepwise approach was used to further select the best model. A lncRNA-based prognostic risk score was calculated based on a linear combination of regression coefficients from the multivariate Cox-regression model $(\beta)$ and its expression levels $(10,11)$.

$$
\text { Prognostic index }=\sum_{i=1}^{N} \operatorname{Exp}_{i} \times \beta_{i}
$$

The Rpackage was used to find the optimal median threshold. According to the optimal median threshold, the survival data of 1,076 patients with BRCA were divided into low-risk and high-risk groups. Kaplan-Meier (KM) survival curves were generated to assess OS in low-risk or high-risk cases and time-dependent receiver operating characteristic (ROC) curve analysis was performed to calculate area under the curve (AUC) values to assess the predictive power of the model (14). Subsequently, we applied the model to patients with

TABLE 2 | Thirteen prognosis-related IncRNAs obtained based on univariate Cox regression analysis $(P<0.01)$.

\begin{tabular}{lccc}
\hline Name & HR & $\boldsymbol{z}$ & $\boldsymbol{p}$-value \\
\hline LINC02037 & 1.243690056 & 4.120958497 & $3.77 \mathrm{E}-05$ \\
LINC01234 & 1.154170798 & 3.811036114 & $1.38 \mathrm{E}-04$ \\
LINC00668 & 1.105563899 & 3.700969179 & $2.15 \mathrm{E}-04$ \\
LINC01456 & 1.132314635 & 3.598894647 & $3.20 \mathrm{E}-04$ \\
LINC01592 & 1.238001411 & 3.465525511 & $5.29 \mathrm{E}-04$ \\
LINC02418 & 1.154200697 & 3.230285935 & $1.24 \mathrm{E}-03$ \\
LINC01854 & 1.221094553 & 2.881476012 & $3.96 \mathrm{E}-03$ \\
C6orf99 & 1.225252162 & 2.837096926 & $4.55 \mathrm{E}-03$ \\
LINC00536 & 1.117384364 & 2.763536856 & $5.72 \mathrm{E}-03$ \\
LINC01224 & 0.916544112 & -2.70404596 & $6.85 \mathrm{E}-03$ \\
LINC02408 & 1.19605941 & 2.681361271 & $7.33 \mathrm{E}-03$ \\
LINC00377 & 0.748711948 & -2.67297483 & $7.52 \mathrm{E}-03$ \\
LINC01574 & 1.145235673 & 2.589564334 & $9.61 \mathrm{E}-03$
\end{tabular}

A

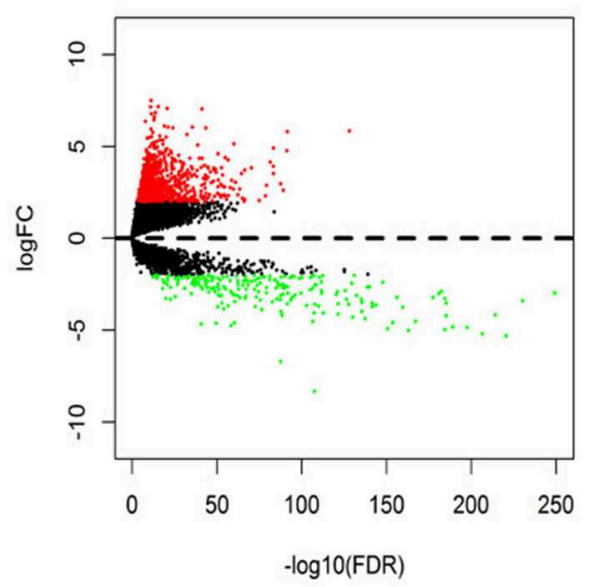

B

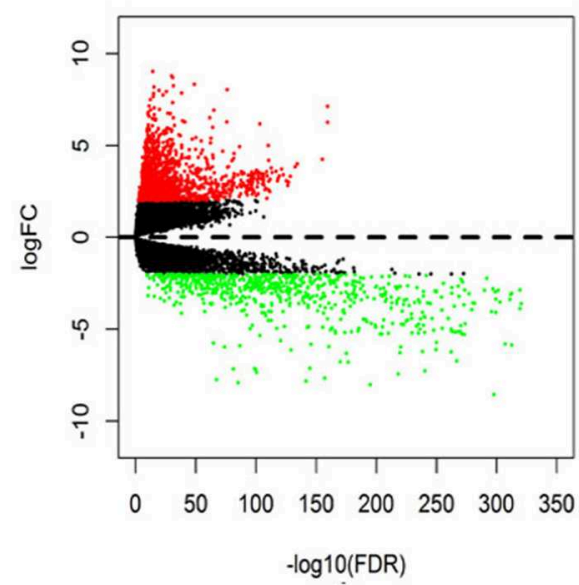

FIGURE 1 | The volcano diagram about differentially expresses IncRNAs (A) and mRNAs (B) between breast cancer tissue and normal tissue samples. Red dots represent up-regulated RNA and green dots represent down-regulated RNA. 
stage I, II, III, and Her2 positive BRCA to test the sensitivity and effectiveness of the model for survival prediction. In addition, we compared the predictive performance of 7-lncRNA model with traditional clinical risk factors (including age, TNM, stage, ER, PR, and HER2 status) by univariate and multivariate Cox analysis. First of all, univariate Cox analysis found factors closely related to the prognosis of patients. Then, the effects of many factors on survival time were analyzed at the same time, and the independent prognostic factors could be used to evaluate the survival of patients. $P<0.05$ was used as the cutoff condition to verify the ability of the model to evaluate the prognosis and sensitivity of patients.

\section{Co-expression Method Predicts IncRNA-Related mRNAs}

To better explore the function of the relevant lncRNAs in the risk assessment model, the related mRNAs were predicted by co-expression methods based on the Pearson correlation. The related mRNAs were screened for functional enrichment analysis according to $|\mathrm{COR}|>0.25, p<0.05$. In addition, the IncRNA-mRNA co-expression network was visualized using Cytoscape.

\section{GO and KEGG Analysis of IncRNA-Related mRNA}

To understand the underlying biological pathways between lncRNA and the related mRNAs, the database for annotation, visualization, and integrated discovery (DAVID) (http://david. abcc.ncifcrf.gov/) was used to perform functional enrichment analysis (15). Subsequently, lncRNA-related mRNAs were analyzed using the gene ontology (GO) database (http://www. geneontology.org). Finally, significantly enriched GO terms were selected to analyze their biological function. The Kyoto Encyclopedia of Genes and Genomes (KEGG; http://www.kegg. $\mathrm{jp} /$ ) was used to perform the pathway enrichment analysis.

\section{RESULTS}

\section{Differentially Expressed IncRNAs and mRNAs in BRCA Patients}

In this study, 1,208 samples were downloaded from the TCGA database and were used to identify differentially-expressed

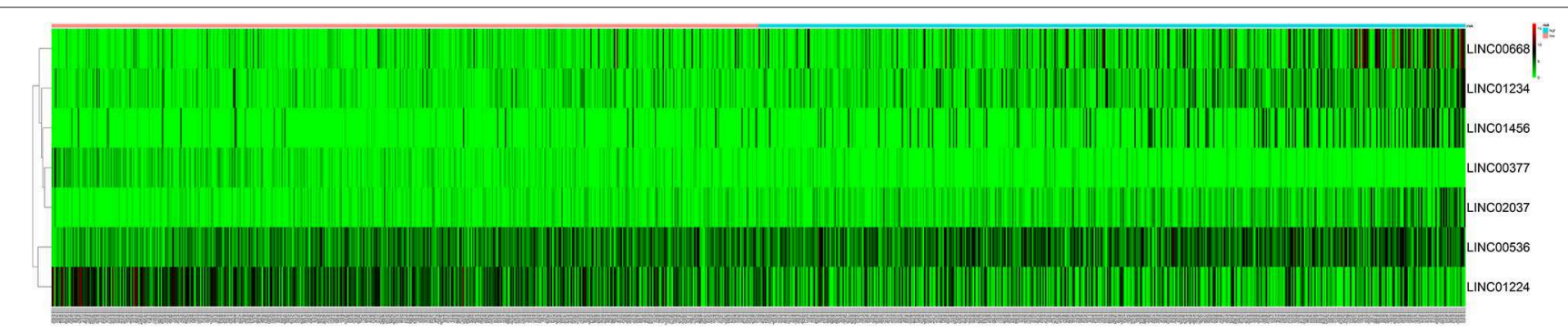

FIGURE 2 | The heatmap of 7 independent breast cancer-related prognostic IncRNAs in the model. The color from green to red indicates a trend from low to high expression.

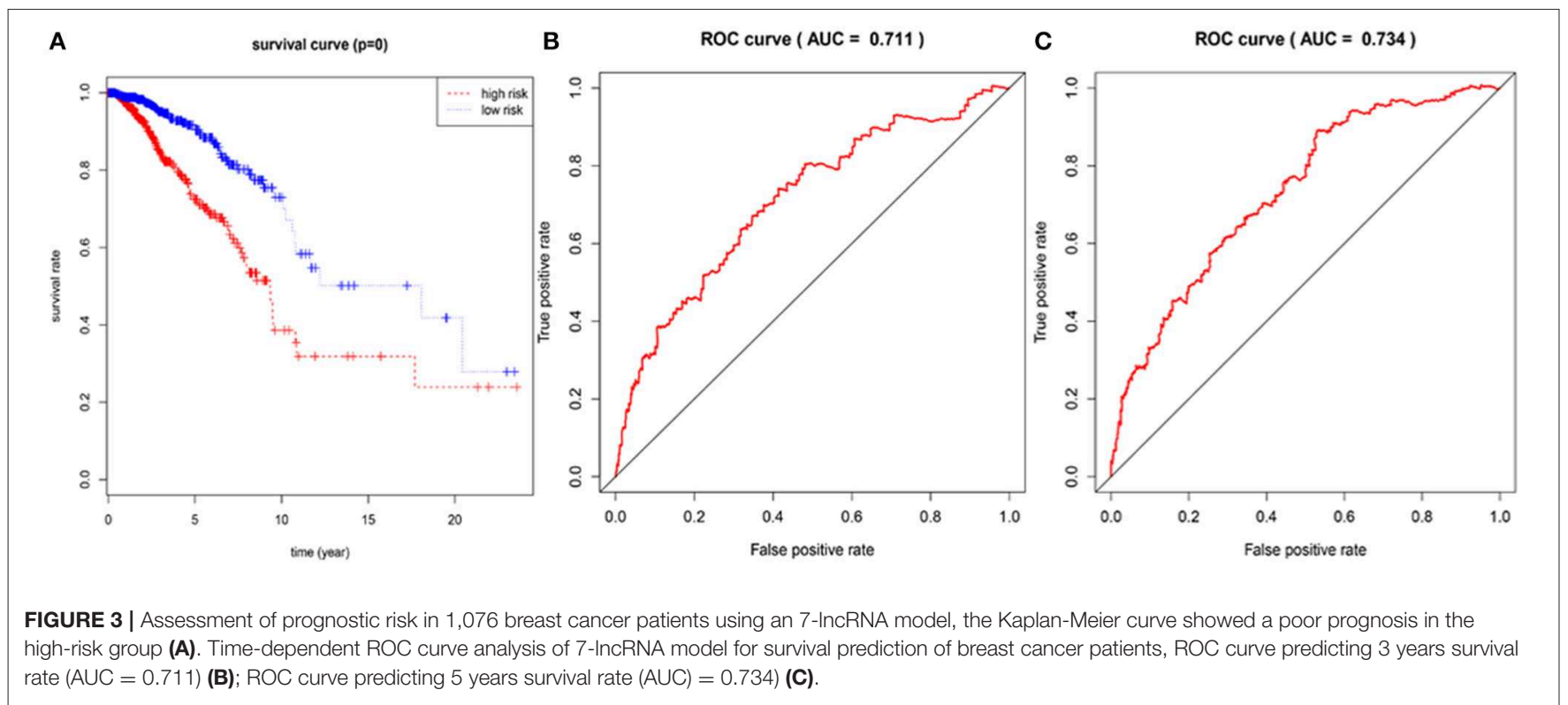


lncRNAs and mRNAs in BRCA patients, We analyzed the specific baseline clinical characteristic of 1,076 BRCA patients presented in Table 1. A total of 1,059 differentially expressed lncRNAs were obtained in accordance with $|\log \mathrm{FC}|>2$ and $p<0.01$. This included 842 upregulated lncRNAs and 217 downregulated lncRNAs (Figure 1A), and 2,138 differentially-expressed mRNAs included 1,375 upregulated mRNAs and 763 downregulated mRNAs (Figure 1B).

\section{Derivation of IncRNA Prognostic Model}

After excluding lncRNA without specific names and lack of corresponding studies, a total of 282 differentially-expressed

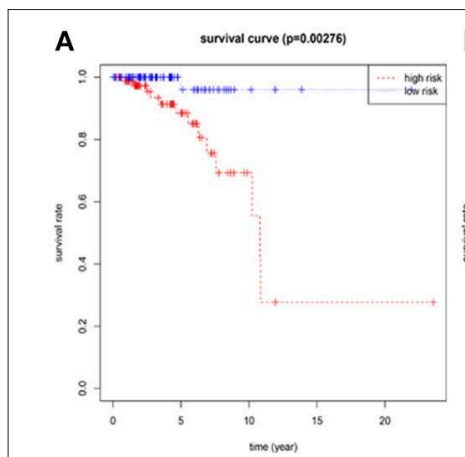

E

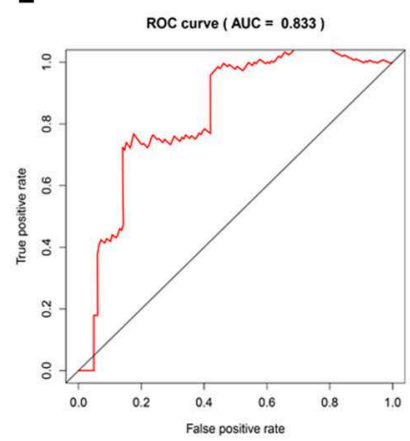

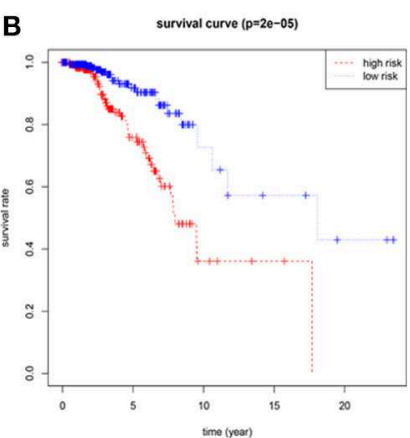

$\mathbf{F}$

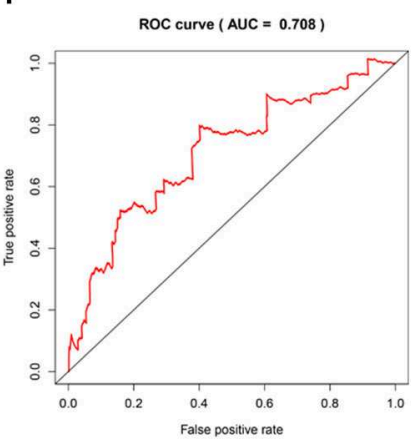

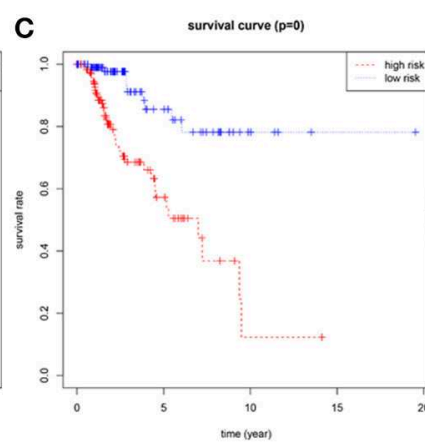

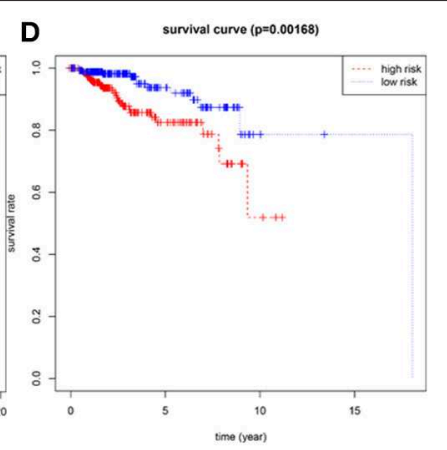

G

ROC curve ( AUC $=0.773)$

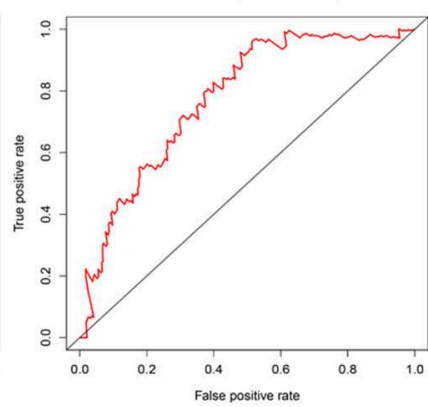

H

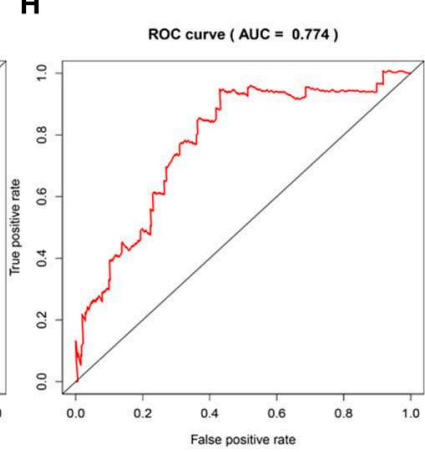

FIGURE 4 | Verification the specificity and sensitivity of the 7-IncRNA prognostic model. The Kaplan-Meier curve of patients with stage I, stage II, stage III, and Her2-positive BRCA (A-D); the ROC curve of the model at 3 years of OS with stage I, stage II, stage III, and Her2-positive BRCA, the AUC values were 0.883, 0.708, $0.773,0.774(\mathbf{E}-\mathrm{H})$.

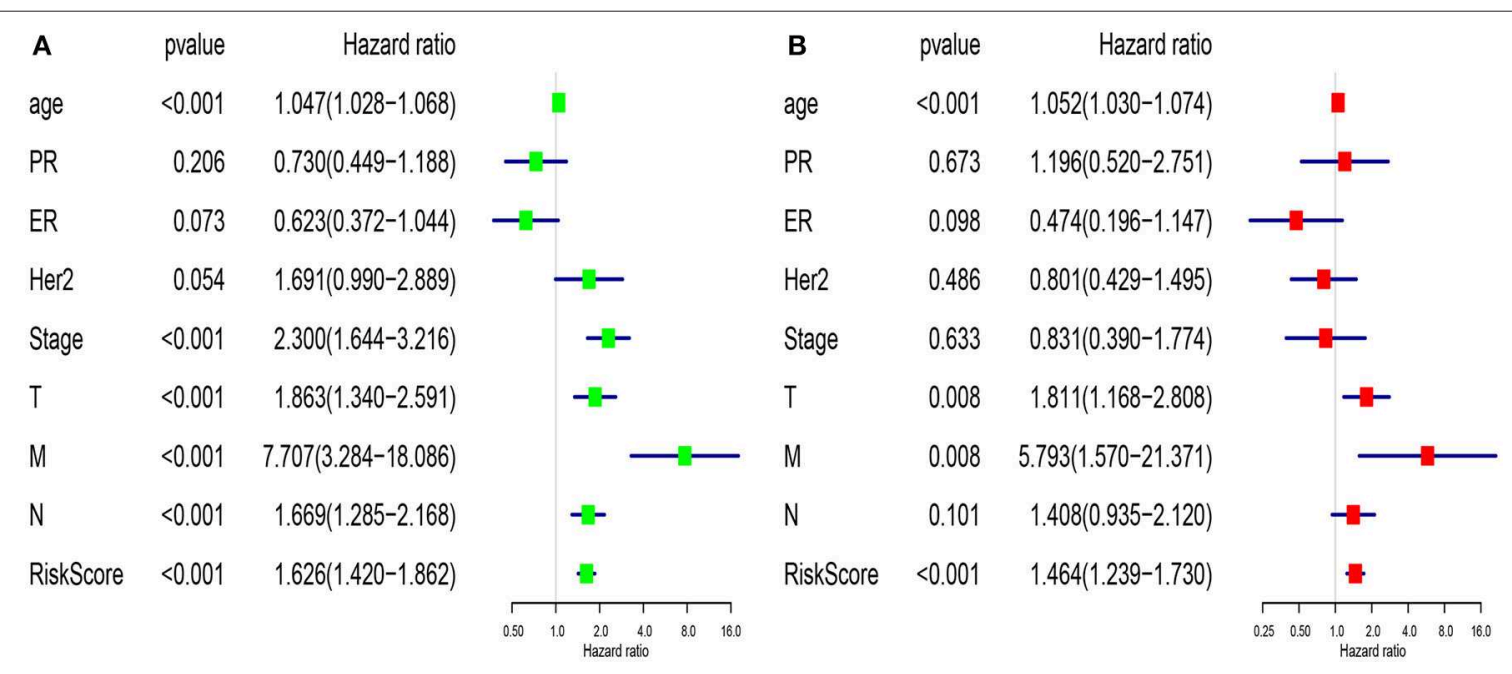

FIGURE 5 | Univariate (A) and multivariate (B) analysis of clinic pathologic factors for overall survival of breast cancer patients from TCGA. 
lncRNAs remained for further study. Firstly, we performed a univariate Cox-regression analysis to study the correlation between differentially-expressed lncRNA and OS of BRCA patients. With a $p<0.01$ as an identification standard, a total of 13 lncRNAs were obtained, which were significantly associated with OS in BRCA patients (Table 2). Subsequently, based on the primary screening using univariate Cox-regression analysis, we obtained seven lncRNAs that were used to construct a predictive model by performing stepwise multivariate Cox-regression analysis. They were LINC00377, LINC00536, LINC01224, LINC00668, LINC01234, LINC02037, and LINC01456 and the cluster dendrogram for these lncRNA is shown in Figure 2. The predictive model was characterized by the linear combination of the expression levels of the seven lncRNAs weighted by their relative coefficients from the multivariate Cox regression as follows:

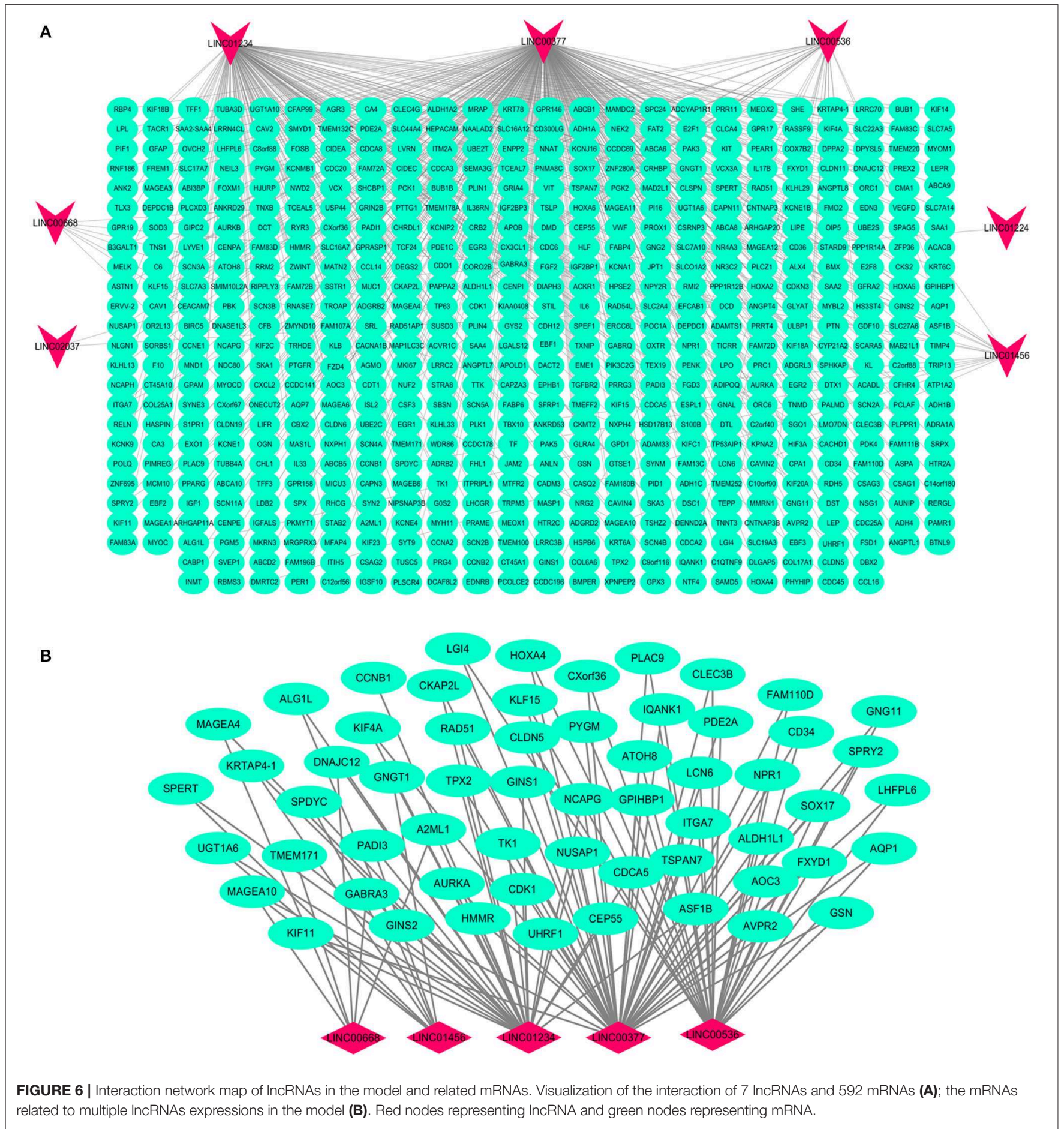


Prognostic index $(\mathrm{PI})=(-0.2611 \times$ expression level of LINC00377 $)+(0.0960 \times$ expression level of LINC00536 $)+$ $(-0.0966 \times$ expression level of LINC01224) $+(0.0738 \times$ expression level of LINC00668) $+(0.1014 \times$ expression level of LINC01234 $)+(0.2020 \times$ expression level of LINC02037) + (0.0627 $\times$ expression level of LINC01456).

Of these seven lncRNAs obtained by Cox-regression analysis, five (LINC00536, LINC00668, LINC01234, LINC02037, and LINC01456) showed positive coefficients, suggesting that these lncRNAs have a higher risk and their expression corresponds to the shorter OS in BRCA patients. In addition, the risk prediction correlation analysis between the seven lncRNAs is presented in Supplementary Figure 1. At the same time, the remaining two lncRNAs (LINC00377 and LINC01224) showed negative coefficients. Although the risk associated with these two lncRNAs is not higher, they are still important links in the prognosis model. These seven lncRNAs together constitute a prognostic model for patients with BRCA.
In the 1,076 BRCA patients, the median of the prognostic score was obtained as the grouping threshold by calculating the risk scores for the expression of the seven lncRNAs. With a median PI as the group threshold, 538 patients with a prognostic score above the PI threshold were classified as high risk, while 538 patients below the PI threshold were assigned to the low-risk group. We found that Kaplan-Meier survival curve analysis of the high-risk and low-risk groups based on the prognostic risk model constructed by the seven IncRNAs showed that the overall survival rate of the high-risk group was lower, and the difference between the two groups was statistically significant (Figure 3A). Subsequently, the prognostic ability of the 7-lncRNA prognostic model was evaluated by calculating the AUC of the timedependent ROC curve. Based on earlier results of the RUC curve, the higher the AUC, the better is the prediction performance of the model. For 3- and 5-year survival times, the AUC of the 7-lncRNA BRCA patient prognostic model was 0.711 and 0.734 , respectively, indicating that the predictive model is highly sensitive and specific (Figures 3B,C).

TABLE 3 | Functional enrichment analysis of IncRNA-related mRNAs.

\begin{tabular}{|c|c|c|c|}
\hline Category & Term & Count & $P$-Value \\
\hline \multirow[t]{8}{*}{ Biological Processes } & Cell division & 45 & $1.13 \mathrm{E}-15$ \\
\hline & Mitotic nuclear division & 37 & $5.70 \mathrm{E}-15$ \\
\hline & Positive regulation of cell proliferation & 32 & 4.11E-05 \\
\hline & Cell proliferation & 26 & $1.49 \mathrm{E}-04$ \\
\hline & Cell adhesion & 26 & $3.81 \mathrm{E}-03$ \\
\hline & Cell surface receptor signaling pathway & 19 & $1.90 \mathrm{E}-03$ \\
\hline & DNA replication & 17 & 2.07E-05 \\
\hline & G2/M transition of mitotic cell cycle & 16 & $1.86 \mathrm{E}-05$ \\
\hline \multirow[t]{7}{*}{ Molecular Function } & ATP binding & 61 & $1.08 \mathrm{E}-02$ \\
\hline & Calcium ion binding & 35 & $5.20 E-03$ \\
\hline & $\begin{array}{l}\text { Transcriptional activator activity, RNA polymerase II core promoter proximal region } \\
\text { sequence-specific binding }\end{array}$ & 16 & 4.87E-03 \\
\hline & ATPase activity & 15 & 1.19E-03 \\
\hline & Heparin binding & 14 & $1.02 \mathrm{E}-03$ \\
\hline & Transporter activity & 14 & $7.65 \mathrm{E}-03$ \\
\hline & Microtubule motor activity & 12 & $2.41 \mathrm{E}-05$ \\
\hline \multirow[t]{5}{*}{ Cellular component } & Plasma membrane & 148 & $1.38 \mathrm{E}-02$ \\
\hline & Cytosol & 122 & $1.36 \mathrm{E}-02$ \\
\hline & Extracellular region & 79 & $2.15 E-05$ \\
\hline & Integral component of plasma membrane & 67 & $2.85 E-04$ \\
\hline & Extracellular space & 64 & $3.66 \mathrm{E}-04$ \\
\hline
\end{tabular}

Enrichment analysis of biological processes, molecular function, and cellular component $(P<0.05)$. 
To confirm the validity and sensitivity of the 7-lncRNA model for predicting survival, we applied the model to risk assessment in patients with stage I, stage II, stage III, and HER2 positive BRCA. Patients were divided into high-risk and low-risk groups using a median risk score (value $=0.965$ ). The Kaplan-Meier curve results showed that the high-risk groups of patients with stage I, stage II, stage III, and Her2-positive BRCA were closely associated with poor prognosis (Figures 4A-D). In addition, the ROC curve indicated that the AUC values of the model were $0.883,0.708,0.773,0.774$ at 3 years of OS (Figures $4 \mathbf{E}-\mathbf{H}$ ), indicating that the 7-lncRNA model we constructed had certain specificity and sensitivity in evaluating the prognosis of patients with BRCA.

\section{Comprehensive Assessment of Model Predictive Performance and Routine Clinical Risk Factors}

We compared the predictive performance of the 7 -lncRNA model with conventional clinical risk factors, including age,
TNM, Stage, ER, PR, and HER2 status. Univariate analysis found that age, Stage, TNM stage, and predictive performance of the 7-lncRNA model were closely related to prognosis (Figure 5A). Further multivariate analysis found that predictive performance of age, T, M, and 7-lncRNA models could be used as independent prognostic factors to assess patient outcomes (Figure 5B).

\section{Functional Assessment of IncRNA-Related mRNA}

Based on the BRCA-related lncRNA and mRNA expression data from the TCGA database, co-expression analysis was performed using the Pearson correlation with $|\mathrm{COR}|>0.25$ and $p<0.05$ as the cutoff. A total of $592 \mathrm{mRNAs}$ were found to be closely related to the 7 lncRNAs (Figure 6). The functions of the lncRNA-related mRNAs were determined using DAVID bioinformatics resources 6.8. The results of GO analysis mainly include Biological Process (BP), Molecular Function (MF), and Cellular Component (CC) (Table 3). We selected the most significant 10 enrichment results in the 3 parts for analysis. The process of enrichment in BP

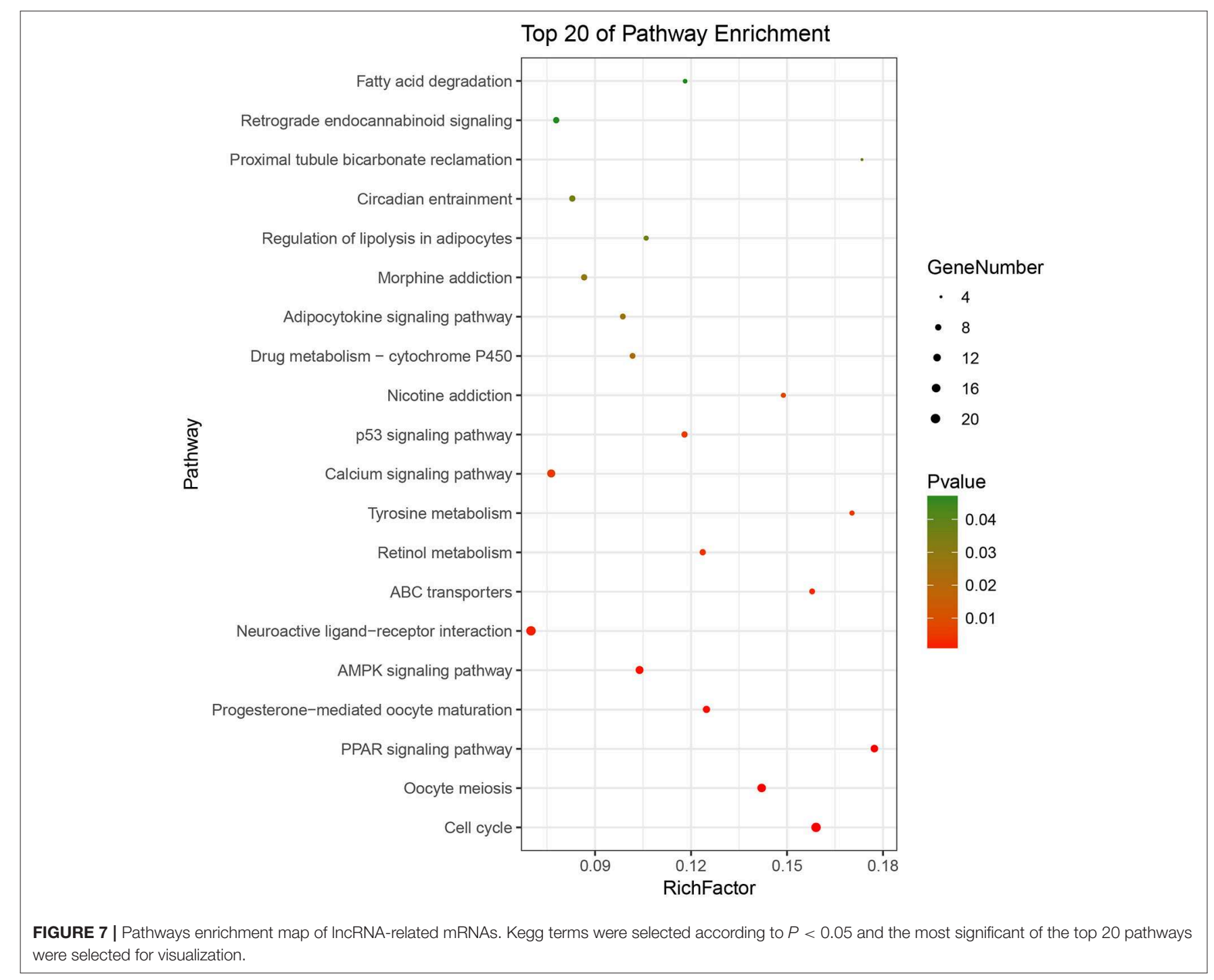




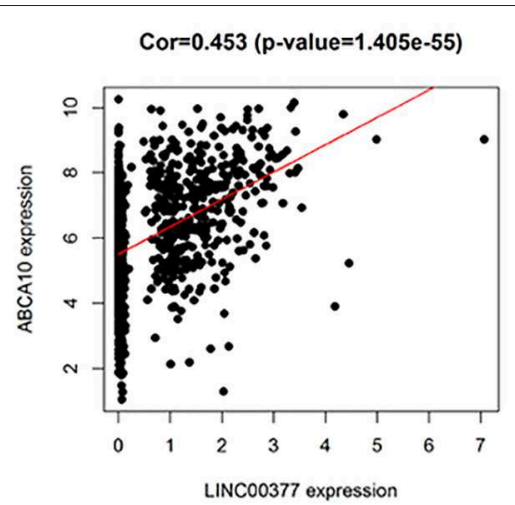

Cor $=0.27(p-$ value $=1.727 e-19)$

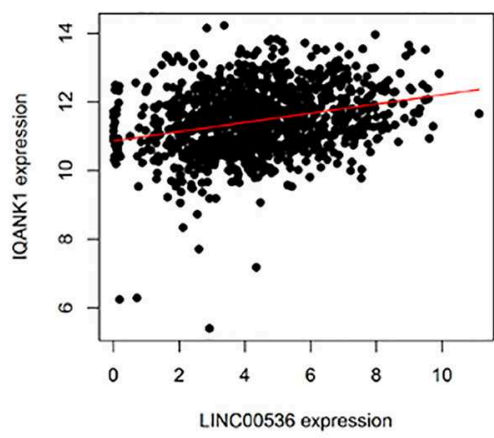

Cor $=0.252(p-$ value $=4.211 e-17)$

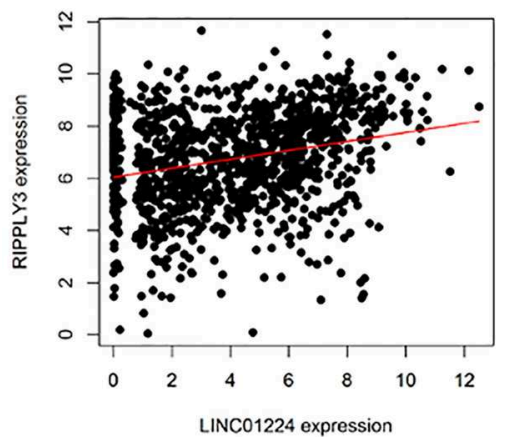

Cor $=0.402(p-$ value $=5.162 e-43)$

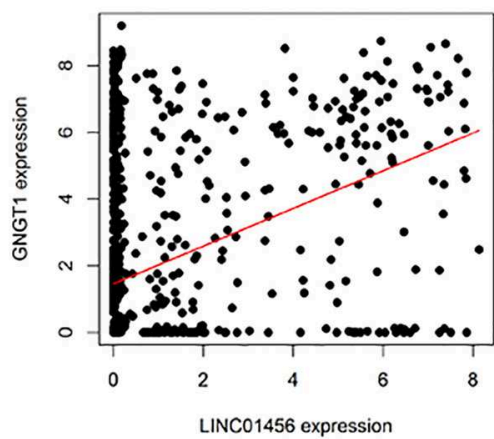

Cor $=-0.29(p-$ value $=3.09 e-22)$

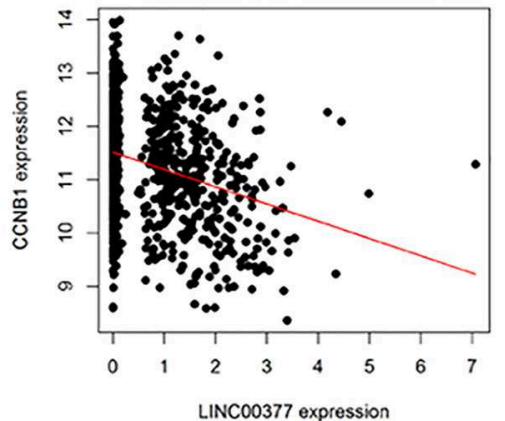

Cor $=0.282(p-$ value $=4.742 e-21)$

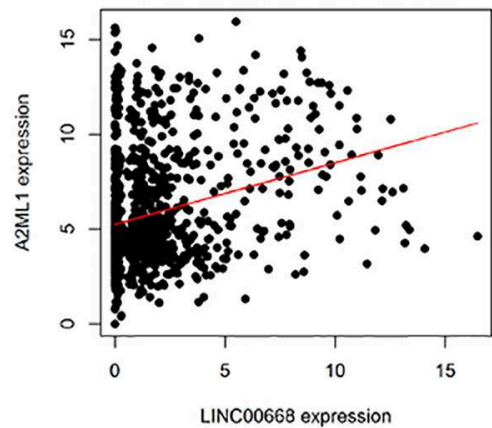

Cor $=-0.356(p-$ value $=1.674 e-33)$

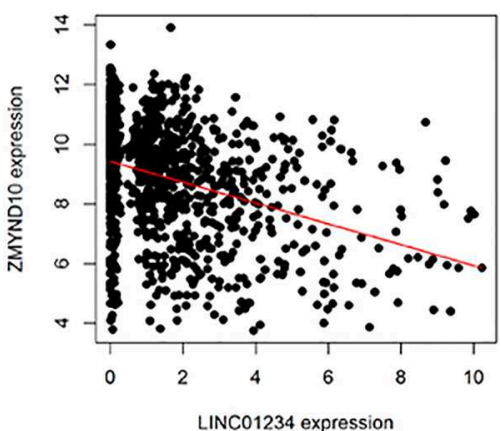

Cor $=0.279(p-$ value $=1.012 \mathrm{e}-20)$

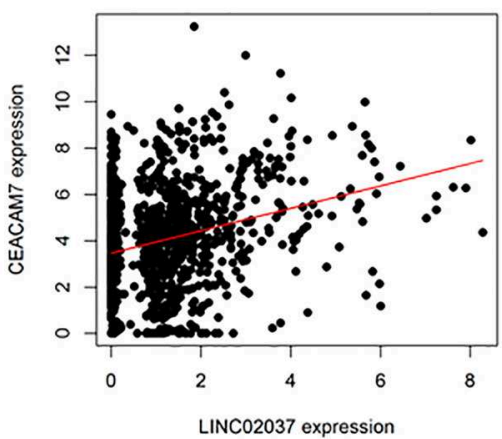

Cor $=-0.309(p-v a l u e=2.886 e-25)$

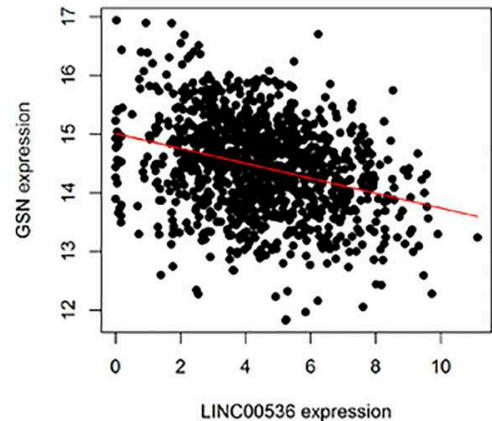

Cor $=-0.27$ (p-value $=1.771 \mathrm{e}-19)$

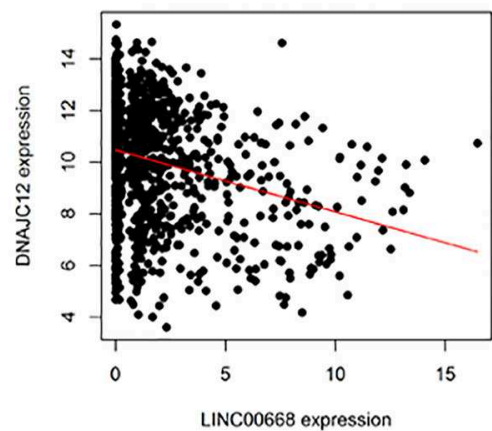

Cor $=0.406(p-$ value $=6.173 e-44)$

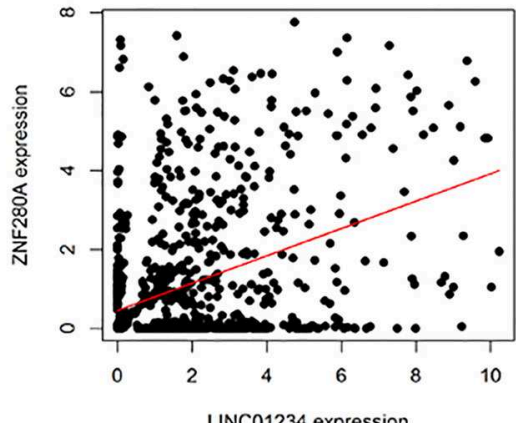

FIGURE 8 | The relationship between 7 IncRNAs and related mRNAs (only the mRNAs that are most positively and negatively correlated with 7 IncRNAs are listed according to the correlation coefficient). 
mainly includes cell division, cell proliferation, cell adhesion, and DNA replication, processes that are closely related to the growth and proliferation of tumor cells. The characteristics of enrichment in MF are mainly ATP binding, calcium-ion binding, chromatin binding, and protein-kinase binding, and those related to CC are plasma membrane, cytosol, integral component of plasma membrane, and the extracellular region. Five hundred ninety-two mRNAs were mainly enriched in 20 signaling pathways (Figure 7), including cell cycle, oocyte meiosis, and other cell division and proliferation pathways; and cancer-related signaling pathways, such as PPAR signaling pathway, neuroactive ligand-receptor interaction, and p53 signaling pathway.

In addition, we identified up-regulated and down-regulated mRNA with the highest correlation coefficient with 7 lncRNAs, and obtained a total of 11 mRNAs, including $A B C A 10, C C N B 1$, GSN, IQANK1, A2ML1, DNAJC12, RIPPLY3, ZMYND10, ZNF280A, GNGT1, and CEACAM7 (Figure 8).

\section{DISCUSSION}

BRCA is still one of the deadliest malignant tumors worldwide (16). Due to its complex molecular and cellular heterogeneity, the efficacy of existing breast cancer risk prediction models is unsatisfactory (17). High recurrence rate of breast cancer is one of the causes of high mortality. Therefore, in order to reduce mortality and improve the prognosis of BRCA, there is a need to construct a new breast cancer risk prediction model for clinical use. Clinicians should be able to develop individualized treatment plans for BRCA patients, establish strategies for prevention and early detection of BRCA recurrence, more frequently track highrisk populations, and perform regular clinical examinations for early diagnosis and recurrence of BRCA based on the predictions of the model.

In this study, BRCA-related differentially-expressed lncRNAs and mRNAs were obtained based on high-throughput RNA sequencing and clinical data of BRCA patients from the TCGA database. Subsequently, univariate and multivariate Cox analysis was performed to establish a risk model for predicting BRCA prognosis. Finally, BRCA prognostic risk prediction model was constructed using seven lncRNAs (LINC00377, LINC00536, LINC01224, LINC00668, LINC01234, LINC02037, and LINC01456). Applying the prognostic model to the TCGA BRCA dataset, breast cancer patients can be divided into highrisk and low-risk groups. The three- and 5-year AUC values for the time-dependent ROC curve were 0.771 and 0.734 , respectively, indicating that the 7 -lncRNA model has a good performance insurvival prediction. By exploring the correlation between differentially-expressed lncRNAs and mRNAs, lncRNArelated mRNAs were identified to further study the function of the 7 lncRNAs and the molecular mechanisms involved in breast cancer progression.

In the current study, among these 7 lncRNAs, LINC00668, LINC01234, and LINC01456 have been shown to play a role in the pathogenesis and prognosis of cancer. Zhao et al. (18) showed that in laryngeal squamous cell carcinoma, the expression levels of LINC00668 were associated with age, pathological differentiation degree, $\mathrm{T}$ stage, clinical stage, and cervical lymph node metastasis, and using a series of bioinformatics tools and in vitro experiments, proved that knockdown of LINC00668 can inhibit the proliferation, migration, and invasion ability of laryngeal squamous cell carcinoma cells. Zhang et al. (19) found that the expression of LINC00668 was negatively correlated with miR-297 expression in oral squamous cell carcinoma, and further found that LINC00668 promoted oral squamous cell carcinoma tumorigenesis via miR-297/VEGFA axis. In addition, Zhang et al. (20) found that knockdown of LINC00668 significantly inhibited the proliferation of gastric cancer cells in vitro and in vivo, and the significant increase in expression was associated with gastric cancer outcomes and prognosis. In our study, we found that the expression of LINC00668 is associated with A2ML1 and DNAJC12; of which A2ML1 has been shown to be closely related to the treatment of lung squamous cell carcinoma and can be used as a potential prognostic biomarker (21). Bubnov et al. (22) used genome-wide microarray Sentrix HumanWD6V3 BeadChip (Illumina) to analyze gene expression pattern in 15 invasive adenocarcinoma samples and 15 healthy breast tissue samples, and found that DNAJC12, a member of the HSP40/DNAJ family, was significantly elevated. In addition, De Bessa et al. (23) found that DNAJC12 is an estrogen target gene, its expression can be used as a marker of the ER activity, and that it may have a predictive value in response to hormonal therapy.

LINC01234 has been shown to be significantly associated with cancer treatment and prognosis in colon, gastric, and breast cancer (24-26). Chen et al. (27) found that LINC01234 expression was significantly upregulated in gastric cancer tissue and was associated with larger tumor size, advanced TNM stage, lymph node metastasis, and shorter survival. Furthermore, knockdown of LINC01234 induced apoptosis, arrested growth, and inhibited tumorigenesis in mouse xenografts. In our study, LINC01234 was found to be associated with ZMYND10 and ZNF280A. ZMYND10, a candidate tumor suppressor gene, is frequently downregulated in nasopharyngeal carcinoma and many other tumors like gastric cancer, due to hypermethylation of the promoter (28). Functional evidence suggests that the ZMYND10 gene inhibits tumor growth in animal experiments (29). According to reports, LINC01456 is a risk factor in ovarian cancer and is involved in the progression of ovarian cancer (30). In our study, we found a positive correlation between GNGT1 and LINC01456 expression.

So far, no studies have reported any association between LINC00377, LINC00536, LINC01224, and LINC02037, and cancer. However, in our study, LINC00377 was found to be associated with expression of $A B C A 10$ and CCNB1. Ho et al. (31) found that $A B C A 10$ is involved in the pathogenesis of osteosarcoma, while Elsnerova et al. (32) found that the expression level of $A B C A 10$ was significantly associated with progression-free survival in ovarian cancer. CCNB1 belongs to the highly conserved cyclin family and is significantly overexpressed in various cancer types. Ding et al. (33), showed that CCNB1 had a significant predictive power in distant metastasis free survival, disease free survival, recurrence free survival, and overall survival of ER+ breast cancer patients. They also found that CCNB1 was closely associated with hormone 
therapy resistance. LINC00536 was found to be associated with expression of GSN and IQANK1, a ubiquitous actin filamentcleaving protein and a well-known downregulated target in breast tumors (34). GSN overexpression studies in MDA-MB231 and MCF-7 cells indicated that increased expression of GSN can result in changes in cell proliferation and cell-cycle progression (35). In addition, Chang et al. (36) showed that LINC01224 is associated with the expression of RIPPLY3, LINC02037 is associated with the expression of CEACAM7, and $C E A C A M 7$ is found to be a potential prognostic biomarker for colorectal cancer.

The use of the TCGA database broadens the range of models for cancer survival prediction. Compared with the previously constructed breast cancer lncRNA prognosis model $(37,38)$, the patient's sample data in the TCGA database is large, and the clinical information is complete, and there is complete prognosis survival data of breast cancer patients. The ROC curve can be used to assess the specificity and sensitivity of the model (AUC $>0.7$ indicates that the model has good sensitivity). The 7-lncRNA prognostic model we developed has the potential to predict the prognosis of patients with BRCA and is specific and sensitive. In addition, whether univariate or multivariate Cox-regression analysis, the predictive performance of the 7-lncRNA model we constructed can be a good assessment of prognosis, further indicating the evaluation value of the model. In addition, as the lncRNAs used in the model have a predictive effect on the prognosis of patients with BRCA, further experimental studies can be conducted to investigate the role of these lncRNAs in the pathogenesis of BRCA in order to provide new ideas and insights for treatment. However, current research still has some limitations, we attempted to validate the predictive performance of the 7-lncRNA model in other large breast cancer data sets. Unfortunately, due to the limitations of the clinical mutation information of breast cancer and patient prognosis information, we did not find a data set that met the verification requirements. So it is

\section{REFERENCES}

1. Kim YA, Cho H, Lee N, Jung SY, Sim SH, Park IH, et al. Doxorubicininduced heart failure in cancer patients: a cohort study based on the Korean National Health Insurance Database. Cancer Med. (2018) 7:6084-92. doi: 10.1002/cam4.1886

2. Yang B, Chou J, Tao Y, Wu D, Wu X, Li X, et al. An assessment of prognostic immunity markers in breast cancer. NPJ Breast Cancer. (2018) 4:35. doi: 10.1038/s41523-018-0088-0

3. Senkus E, Kyriakides S, Ohno S, Penault-Llorca F, Poortmans P, Rutgers E, et al. Primary breast cancer: ESMO clinical practice guidelines for diagnosis, treatment and follow-up. Ann Oncol. (2015) 26(Suppl. 5), v8-30. doi: $10.1093 /$ annonc/mdv298

4. Lou W, Liu J, Ding B, Xu L, Fan W. Identification of chemoresistanceassociated miRNAs in breast cancer. Cancer Manag Res. (2018) 10:4747-57. doi: 10.2147/CMAR.S172722

5. Tian T, Wang M, Zhu Y, Zhu W, Yang T, Li H, et al. Expression, clinical significance, and functional prediction of MNX1 in breast cancer. Mol Ther Nucleic Acids. (2018) 13:399-406. doi: 10.1016/j.omtn.2018.09.014

6. Wang Z, Gerstein M, Snyder M. RNA-Seq: a revolutionary tool for transcriptomics. Nat Rev Genet. (2009) 10:57-63. doi: 10.1038/nrg2484 necessary to propose effective strategies such as including longer follow-up duration to validate the results and multiple regression modeling methods to improve the accuracy of the model.

\section{CONCLUSION}

We constructed a 7-lncRNA prognostic model to reliably predict the prognosis of patients with BRCA, and these lncRNAs may play a role in the carcinogenesis of BRCA. Further functional studies are needed to elucidate the molecular mechanisms behind the roles of these lncRNAs in BRCA.

\section{DATA AVAILABILITY STATEMENT}

This manuscript contains previously unpublished data. The name of the repository and accession number are not available.

\section{AUTHOR CONTRIBUTIONS}

CS, HL, and CG conceived and designed the study. LL, JZ, and JY performed data analysis. CL, CZ, and FF contributed analysis tools. HL and CG wrote the paper.

\section{FUNDING}

This work was supported by the grants from National Natural Science Foundation of China (81673799) and National Natural Science Foundation of China Youth Fund (81703915).

\section{SUPPLEMENTARY MATERIAL}

The Supplementary Material for this article can be found online at: https://www.frontiersin.org/articles/10.3389/fonc. 2019.01348/full\#supplementary-material

Figure S1 | The risk prediction correlation analysis between the seven IncRNAs.

7. Yan X, Hu Z, Feng Y, Hu X, Yuan J, Zhao SD, et al. Comprehensive genomic characterization of long non-coding RNAs across human cancers. Cancer Cell. (2015) 28:529-40. doi: 10.1016/j.ccell.2015.09.006

8. Huarte M. The emerging role of lncRNAs in cancer. Nat Med. (2015) 21:125361. doi: $10.1038 / \mathrm{nm} .3981$

9. Mendell JT. Targeting a long noncoding RNA in breast cancer. $N$ Engl J Med. (2016) 374:2287-9. doi: 10.1056/NEJMcibr1603785

10. Shi X, Tan H, Le X, Xian H, Li X, Huang K, et al. An expression signature model to predict lung adenocarcinoma-specific survival. Cancer Manag Res. (2018) 10:3717-32. doi: 10.2147/CMAR.S159563

11. Long J, Zhang L, Wan X, Lin J, Bai Y, Xu W, et al. A four-gene-based prognostic model predicts overall survival in patients with hepatocellular carcinoma. J Cell Mol Med. (2018) 22:5928-38. doi: 10.1111/jcmm.13863

12. Liu SJ, Lim DA. Modulating the expression of long non-coding RNAs for functional studies. EMBO Rep. (2018) 19:e46955. doi: 10.15252/embr.201846955

13. Tomczak K, Czerwinska $\mathrm{P}$, Wiznerowicz $\mathrm{M}$. The cancer genome atlas (TCGA): an immeasurable source of knowledge. Contemp Oncol (Pozn). (2015) 19:A68-77. doi: 10.5114/wo.2014.47136

14. Yoneda K, Kuwata T, Chikaishi Y, Mori M, Kanayama M, Takenaka $\mathrm{M}$, et al. Detection of circulating tumor cells with a novel microfluidic 
system in malignant pleural mesothelioma. Cancer Sci. (2018) 110:726-33. doi: $10.1111 /$ cas. 13895

15. Jiao X, Sherman BT, Huang da W, Stephens R, Baseler MW, Lane HC, et al. DAVID-WS: a stateful web service to facilitate gene/protein list analysis. Bioinformatics. (2012) 28:1805-6. doi: 10.1093/bioinformatics/bts251

16. Desreux JAC. Breast cancer screening in young women. Eur J Obstet Gynecol Reprod Biol. (2018) 230:208-11. doi: 10.1016/j.ejogrb.2018.05.018

17. Braden AM, Stankowski RV, Engel JM, Onitilo AA. Breast cancer biomarkers: risk assessment, diagnosis, prognosis, prediction of treatment efficacy and toxicity, and recurrence. Curr Pharm Des. (2014) 20:4879-98. doi: 10.2174/1381612819666131125145517

18. Zhao L, Cao H, Chi W, Meng W, Cui W, Guo W, et al. Expression profile analysis identifies the long non-coding RNA landscape and the potential carcinogenic functions of LINC00668 in laryngeal squamous cell carcinoma. Gene. (2018) 687:47-55. doi: 10.1016/j.gene.2018.11.020

19. Zhang CZ. Long intergenic non-coding RNA 668 regulates VEGFA signaling through inhibition of miR-297 in oral squamous cell carcinoma. Biochem Biophys Res Commun. (2017) 489:404-12. doi: 10.1016/j.bbrc.2017.05.155

20. Zhang E, Yin D, Han L, He X, Si X, Chen W, et al. E2F1-induced upregulation of long noncoding RNA LINC00668 predicts a poor prognosis of gastric cancer and promotes cell proliferation through epigenetically silencing of CKIs. Oncotarget. (2016) 7:23212-26. doi: 10.18632/oncotarget.6745

21. Zhang W, Cui Q, Qu W, Ding X, Jiang D, Liu H. TRIM58/cg26157385 methylation is associated with eight prognostic genes in lung squamous cell carcinoma. Oncol Rep. (2018) 40:206-16. doi: 10.3892/or.2018.6426

22. Bubnov V, Moskalev E, Petrovskiy Y, Bauer A, Hoheisel J, Zaporozhan V. Hypermethylation of TUSC5 genes in breast cancer tissue. Exp Oncol. (2012) 34:370-2. Available online at: http://dspace.nbuv.gov.ua/handle/ $123456789 / 139864$

23. De Bessa SA, Salaorni S, Patrao DF, Neto MM, Brentani MM, Nagai MA. JDP1 (DNAJC12/Hsp40) expression in breast cancer and its association with estrogen receptor status. Int J Mol Med. (2006) 17:363-7. doi: 10.3892/ijmm.17.2.363

24. Guo W, Wang Q, Zhan Y, Chen X, Yu Q, Zhang J, et al. Transcriptome sequencing uncovers a three-long noncoding RNA signature in predicting breast cancer survival. Sci Rep. (2016) 6:27931. doi: 10.1038/srep27931

25. Gu J, Li Y, Fan L, Zhao Q, Tan B, Hua K, et al. Identification of aberrantly expressed long non-coding RNAs in stomach adenocarcinoma. Oncotarget. (2017) 8:49201-16. doi: 10.18632/oncotarget.17329

26. Liu JX, Li W, Li JT, Liu F, Zhou L. Screening key long non-coding RNAs in early-stage colon adenocarcinoma by RNA-sequencing. Epigenomics. (2018) 10:1215-28. doi: 10.2217/epi-2017-0155

27. Chen X, Chen Z, Yu S, Nie F, Yan S, Ma P, et al. Long Noncoding RNA LINC01234 functions as a competing endogenous RNA to regulate CBFB expression by sponging miR-204-5p in gastric cancer. Clin Cancer Res. (2018) 24:2002-14. doi: 10.1158/1078-0432.CCR-17-2376

28. Xiao K, Yu Z, Shi DT, Lei Z, Chen H, Cao J, et al. Inactivation of BLU is associated with methylation of Sp1-binding site of BLU promoter in gastric cancer. Int J Oncol. (2015) 47:621-31. doi: 10.3892/ijo.2015.3032
29. Cheng Y, Ho RL, Chan KC, Kan R, Tung E, Lung HL, et al. Antiangiogenic pathway associations of the 3p21.3 mapped BLU gene in nasopharyngeal carcinoma. Oncogene. (2015) 34:4219-28. doi: 10.1038/onc. 2014.353

30. Meng X, Jin-Cheng G, Jue Z, Quan-Fu M, Bin Y, Xu-Feng W. Proteincoding genes, long non-coding RNAs combined with microRNAs as a novel clinical multi-dimension transcriptome signature to predict prognosis in ovarian cancer. Oncotarget. (2017) 8:72847-59. doi: 10.18632/oncotarget. 20457

31. Ho XD, Phung P, Q Le V, H Nguyen V, Reimann E, Prans E, et al. Whole transcriptome analysis identifies differentially regulated networks between osteosarcoma and normal bone samples. Exp Biol Med (Maywood). (2017) 242:1802-11. doi: 10.1177/1535370217736512

32. Elsnerova K, Mohelnikova-Duchonova B, Cerovska E, Ehrlichova M, Gut I, Rob L, et al. Gene expression of membrane transporters: importance for prognosis and progression of ovarian carcinoma. Oncol Rep. (2016) 35:215970. doi: 10.3892/or.2016.4599

33. Ding K, Li W, Zou Z, Zou X, Wang C. CCNB1 is a prognostic biomarker for ER+ breast cancer. Med Hypotheses. (2014) 83:359-64. doi: 10.1016/j.mehy.2014.06.013

34. Gur-Dedeoglu B, Konu O, Bozkurt B, Ergul G, Seckin S, Yulug IG. Identification of endogenous reference genes for qRT-PCR analysis in normal matched breast tumor tissues. Oncol Res. (2009) 17:353-65. doi: 10.3727/096504009788428460

35. Chen ZY, Wang PW, Shieh DB, Chiu KY, Liou YM. Involvement of gelsolin in TGF-beta 1 induced epithelial to mesenchymal transition in breast cancer cells. J Biomed Sci. (2015) 22:90. doi: 10.1186/s12929-015-0197-0

36. Chang J, Huang L, Cao Q, Liu F. Identification of colorectal cancer-restricted microRNAs and their target genes based on high-throughput sequencing data. Onco Targets Ther. (2016) 9:1787-94. doi: 10.2147/OTT.S93338

37. Wang K, Li J, Xiong Y, Zeng Z, Zhang X, Li H. A potential prognostic long noncoding RNA signature to predict recurrence among ER-positive breast cancer patients treated with tamoxifen. Sci Rep. (2018) 8:3179. doi: 10.1038/s41598-018-21581-w

38. Sun J, Chen X, Wang Z, Guo M, Shi H, Wang X, et al. A potential prognostic long non-coding RNA signature to predict metastasis-free survival of breast cancer patients. Sci Rep. (2015) 5:16553. doi: 10.1038/srep16553

Conflict of Interest: The authors declare that the research was conducted in the absence of any commercial or financial relationships that could be construed as a potential conflict of interest.

Copyright (c) 2019 Li, Gao, Liu, Zhuang, Yang, Liu, Zhou, Feng and Sun. This is an open-access article distributed under the terms of the Creative Commons Attribution License (CC BY). The use, distribution or reproduction in other forums is permitted, provided the original author(s) and the copyright owner(s) are credited and that the original publication in this journal is cited, in accordance with accepted academic practice. No use, distribution or reproduction is permitted which does not comply with these terms. 